\title{
Growth of High-Quality GaN Films on Epitaxial AIN/Sapphire Templates
}

\author{
Xiajia Feng* and Yu Yun \\ Guangdong University of Technology, 100 Waihuan Xi Road \\ Panyu District, Guangzhou, China \\ *Email: xjfeng1000@gmail.com
}

\begin{abstract}
High quality GaN films have been successfully grown on multi-AlN/sapphire templates by metal organic chemical vapor deposition system. The Hall mobility and the carrier concentration of $720 \mathrm{~cm}^{2} / \mathrm{Vs}$ and $6.7 \times 10^{16} \mathrm{~cm}^{-3}$ at $300 \mathrm{~K}$, respectively, along with low dislocation density of $4.1 \times 10^{9} \mathrm{~cm}^{-}$ ${ }^{2}$ have been achieved. The X-ray rocking curve full-width at half-maximum were 160 and 290 arcsec for (0004) and (20-24) reflection planes also obtained, respectively. Besides that, the atomic force microscopy images showed smooth surface morphology and a higher intensity near the band edge was also observed by photoluminescence measurement result.
\end{abstract}

\section{Introduction}

GaN, AlN, InN semiconductors are very attractive materials for many applications such as light emitting diodes, photodiodes, high power/frequency/high temperature devices. ${ }^{[1,2]}$ Especially, sapphire has been widely used as a substrate for the growth of GaN films for decades. ${ }^{[3]}$ However, it is difficult to grow high quality GaN films because of lattice constant and thermal expansion coefficient between $\mathrm{GaN}$ and sapphire substrate. ${ }^{[4]}$ Many growth methods have been applied to grow high quality GaN such as using thin AlN or GaN buffer layer deposited at low temperatures on sapphire substrates. ${ }^{[1-6]}$ However, the GaN films grown on sapphire substrates used these methods still showed high dislocation densities and residual stresses. ${ }^{[5]}$ Therefore, we need another method to suppress this problem.

In this study, we report on the growth of high quality AlN film on sapphire substrate at a temperature higher than $1000^{\circ} \mathrm{C}$, which will be a very good template for $\mathrm{GaN}$ epitaxial growth thereafter. When AlN/sapphire template is used as a substrate instead of a low temperature buffer layer as compared to other reports, the overgrown GaN layer is expected to achieve higher crystal quality than those grown on a low temperature buffer layer because the AlN/sapphire template is a single high crystalline quality. Yoshida et al. was the first group that grown GaN on AlN template at high temperature by using the molecular beam epitaxial system with high mobility and peak intensity. ${ }^{[7]}$ However, the result still needs more improvements to be able to satisfy for device development. Thereafter, another group has also grown GaN films on AlN template by using MOCVD system. ${ }^{[8]}$ As similar to the first group, the quality of AlN template was not good with many islands on the surface, resulting low quality GaN films on the top. In this paper, we have clarified the electrical, crystal, optical qualities of GaN films grown on the high quality AlN template. 


\section{Experimental procedure}

The AlN template was grown on 2-inch diameter sapphire substrate by horizontal MOCVD reactor. Trimethylaluminum (TMA) and ammonia $\left(\mathrm{NH}_{3}\right)$ were used as $\mathrm{Al}$ and $\mathrm{N}$ sources, respectively. Ammonia was used as $\mathrm{N}$ source and silane $\left(\mathrm{SiH}_{4}\right)$ was used as the n-type dopant. The substrates were heated at a temperature higher than $1000^{\circ} \mathrm{C}$ and kept it there for $10 \mathrm{~min}$ for stability before growing approximately $1 \mu \mathrm{m}$ thick AlN template. Subsequently, Si-doped approximately $2.5 \mu \mathrm{m}$ thick GaN film was grown on this AlN/sapphire template and compared with another sample that $\mathrm{GaN}$ film was grown with a low temperature $50 \mathrm{~nm} \mathrm{GaN}$ buffer layer on sapphire substrate. The growth conditions were kept the same for two cases. Hall effect measurement was performed using the Van der Pauw technique to measure the mobility. High resolution X-ray diffraction (XRD) measurement was carry out to confirm the crystal quality. The surface morphology was analyzed using AFM technique. For characterizing the optical properties, room-temperature photoluminescence (PL) was measured using the $325 \mathrm{~nm} \mathrm{He}-\mathrm{Cd}$ laser as an excitation light source and GaAs photocathode as a detector.

\section{Results and discussion}

The Hall mobilities of Si-doped GaN films in both cases as a function of the carrier concentration at room temperature. Hall mobilities of Si-doped GaN films grown on AlN/sapphire template with the highest mobility was about $720 \mathrm{~cm}^{2} / \mathrm{Vs}$ and an electron carrier concentration of $6.7 \times 10^{16} \mathrm{~cm}^{-3}$ at $77 \mathrm{~K}$. While the Hall mobilities of GaN films grown on sapphire substrates with low temperature GaN buffer layer at each carrier concentration, all were lower than those grown on high temperature AlN/sapphire templates (figure not showed here). Overall, the crystal quality of GaN films grown on high quality AlN/sapphire templates can be concluded that it is better than those grown on sapphire substrates with conventional low temperature GaN buffer layer.

Thus, the Hall mobilities and carrier concentrations of GaN films grown on AlN/sapphire templates are shown as a function of temperature. The maximum Hall mobility was $1500 \mathrm{~cm}^{2} / \mathrm{Vs}$ with the electron carrier concentration of $3.0 \times 10^{16} \mathrm{~cm}^{-3}$ at $127 \mathrm{~K}$.

The FWHM rocking curve (XRC) measurements was found to be significant difference. Where, GaN films grown on the AlN/sapphire templates were 160 arcsec for the (0004) reflection and 290 arcsec for the (20-24) reflection. In contrast, FWHM XRD of GaN films grown on sapphire substrates with low temperature $\mathrm{GaN}$ buffer layer were 320 and 680 arcsec for (0004) and (20-24) reflections, respectively. Overall, the crystal quality of GaN film grown on the AlN/ sapphire templates was found to be excellent as compared to the other case. The XRC FWHMs of the GaN films grown on AlN/sapphire templates are really better than those grown on low temperature GaN buffer layer in the case of the Hall mobility. Thus, the sample with higher Hall mobility showed better crystal qualities with narrower FWHMs XRC for both reflection planes (0004) and for (20-24) reflection may due to the good crystallinity of AlN/sapphire template. 
Figure 1 shows the AFM images of the GaN film grown on an AlN/sapphire template and the GaN film grown on a sapphire substrate with a low temperature GaN buffer layer. From the Figure 3(a) we can see, a very smooth surface morphology and clear step formation were observed with likely no pit can be found on the surface of the GaN films grown on AlN/sapphire template. Especially, the surface roughness value of the GaN film was just $1.55 \mathrm{~nm}$. The GaN film grown on AlN/sapphire templates exhibited much better morphology compared with those grown on sapphire substrate with low temperature $\mathrm{GaN}$ buffer layer as showed in figure 3(b) with the surface roughness is about $2.91 \mathrm{~nm}$.

Figure 2 shows the room temperature photoluminescence (PL) spectrum of the GaN film grown on an AlN/sapphire template and on a sapphire substrate with a low temperature GaN buffer layer. From the figure, we can see the intensity of deep level emission of both films was almost the same and can be confirm that they are postulated to be associated with Ga vacancies as reported. ${ }^{[9-11]}$ Even though the intensity of yellow luminescence is very high compared with that of the near the band edge emission, the reason may be simply because the PL system has a higher detection efficiency in the yellow luminescence region. In addition, the intensity of the near the band edge emission of GaN films grown on AlN/sapphire template was much higher than that of GaN film which was grown on sapphire substrates with a low temperature GaN buffer layer.

In this paper, we have demonstrated the growth of high quality GaN films by MOCVD, using high quality AlN/sapphire template and compared with GaN films grown on low temperature $\mathrm{GaN}$ buffer layer. Hall mobilities of the $\mathrm{GaN}$ films were $720 \mathrm{~cm}^{2} / \mathrm{Vs}$ with electron carrier concentrations of $6.7 \times 10^{16} \mathrm{~cm}^{-3}$ at $300 \mathrm{~K}$. The FWHM XRCs were 160 and 290 arcsec for the (0004) and (20-24) reflections were also investigated, respectively. AFM showed a very good surface morphology and higher PL intensity near the band edge was observed. Results confirmed that high quality GaN films could be obtained on AlN/sapphire templates.

\section{References}

[1] M Razeghi, "Overview of antimonide based III-V semiconductor epitaxial layers and their applications at the center for quantum devices", The European Physical Journal-Applied Physics 23, 149-205 (2003).

[2] B.T. Tran and H Hirayama, "Growth and Fabrication of High External Quantum Efficiency AlGaN-Based Deep Ultraviolet Light-Emitting Diode Grown on Pattern Si Substrate", Scientific Reports 7, 12176 (2017).

[3] M Razeghi, "Optoelectronic devices based on III-V compound semiconductors which have made a major scientific and technological impact in the past 20 years", IEEE Journal of Selected Topics in Quantum Electronics 6, 1344-1354 (2000).

[4] M Razeghi, "III-nitride optoelectronic devices: from ultraviolet toward terahertz", IEEE Photonics Journal 3, 263-267 (2011).

[5] K. L. Lin, B. T. Tran, C. C. Chung and E. Y. Chang, "Growth of GaN films on circle array patterned Si (111) substrates", Journal of Crystal Growth 401, 648651 (2014)

[6] B. T. Tran, C. H. Ming, K. L. Lin, H. M. Chen, C. C. Wang, C. C. Chen, C. Y. Huang, C. C. Chung and E. Y. Chang, "Growth and Fabrication of GaN Light 
Emitting Diode on Patterned-Sapphire Substrate", ECS Transactions 50, 1-4 (2013).

[7] B. T. Tran, E. Y. Chang, H. D. Trinh, C. T. Lee, K. C. Sahoo, K. L. Lin, M. C. Huang, H. W. Yu, T. T. Luong, C. C. Chung and C. L. Nguyen, "Fabrication and characterization of $\mathrm{n}-\mathrm{In}_{0.4} \mathrm{Ga}_{0.6} \mathrm{~N} / \mathrm{p}-\mathrm{Si}$ solar cell", Solar Energy Materials and Solar Cells 102, 208-211 (2012).

[8] T. T. Luong, Y. T. Ho, B. T. Tran, Y. Y. Woong and E. Y. Chang, "Barrier Strain and Carbon Incorporation-Engineered Performance Improvements for AlGaN/GaN High Electron Mobility Transistors", Chemical Vapor Deposition 21, 33-40 (2015).

[9] F. Lumbantoruan, Y. Y. Wong, W. H. Wu, W. C. Huang, N. M. Shrestra, T. T. Luong, T. B. Tinh and E. Y. Chang, "Investigation of TMAl preflow to the properties of AlN and GaN film grown on Si(111) by MOCVD', IEEE-

ICSE2014 Proc. 2014, Kuala Lumpur, Malaysia.

Doi: 10.1109/SMELEC.2014.6920785

[10] T. T. Luong, B. T. Tran, Y. T. Ho, T. W. Wei, Y. H. Wu, T. C. Yen, L. L. Wei, J. S. Maa, " $2 \mathrm{H}$-silicon carbide epitaxial growth on $c$-plane sapphire substrate using an AlN buffer layer and effects of surface pre-treatments", Electronic Materials Letters 11, 352-359 (2015).

[11] M Razeghi, "Optoelectronic devices based on III-V compound semiconductors which have made a major scientific and technological impact in the past 20 years", IEEE Journal of Selected Topics in Quantum Electronics 6, 1344-1354 (2000).
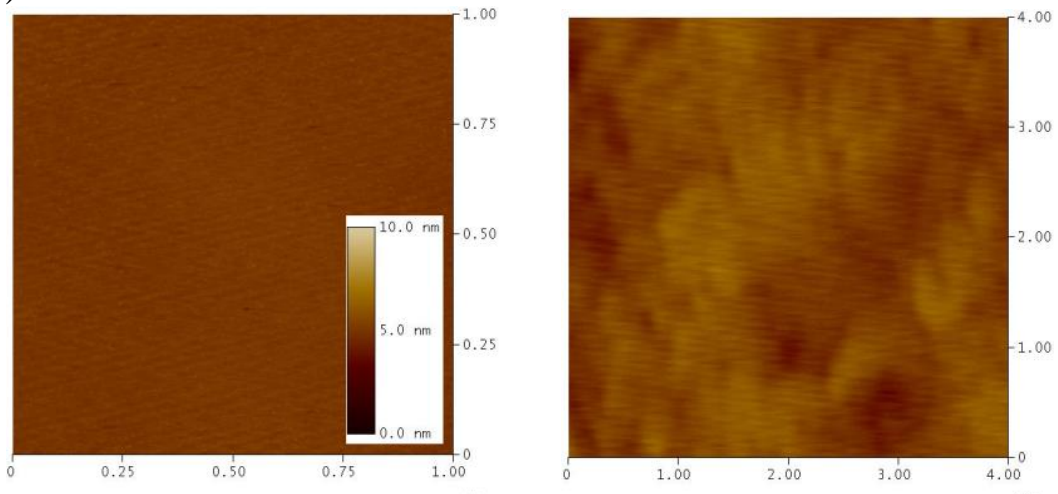

Figure 1 shows the AFM images of the GaN film grown on an AlN/sapphire template (a-left) and the GaN film grown on a sapphire substrate

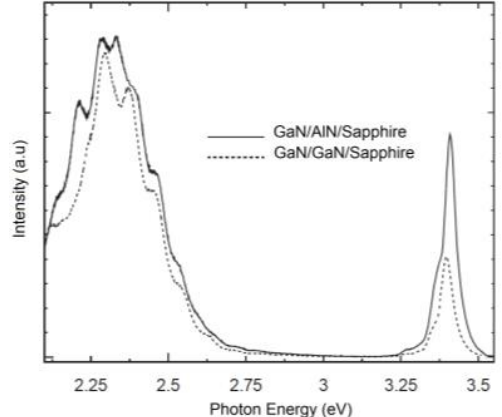

Figure 2 shows the room temperature photoluminescence spectra of the GaN/AlN/sapphire template and on GaN/low temperature GaN/sapphire. 